\title{
Atypical Presentation of Pityriasis Rubra Pilaris: Challenges in Diagnosis and Management
}

\author{
Michael Abrouk, MD; Mio Nakamura, MD; Tian Hao Zhu, MD; Benjamin Farahnik, MD; \\ John Koo, MD; Tina Bhutani, MD
}

\section{PRACTICE POINTS}

- Pityriasis rubra pilaris (PRP) is a rare inflammatory dermatosis of unknown etiology characterized by erythematosquamous salmon-colored plaques with well-demarcated islands of unaffected skin and hyperkeratotic follicles.

- The diagnosis of PRP often can be challenging given the variety of clinical presentations.

To the Editor:

Pityriasis rubra pilaris (PRP) is a rare inflammatory dermatosis of unknown etiology characterized by erythematosquamous salmon-colored plaques with welldemarcated islands of unaffected skin and hyperkeratotic follicles. ${ }^{1}$ In the United States, an incidence of 1 in 3500 to 5000 patients presenting to dermatology clinics has been reported. ${ }^{2}$ Pityriasis rubra pilaris has several subtypes and variability in presentation that can make accurate and timely diagnosis challenging. ${ }^{3-5}$ Herein, we present a case of PRP with complex diagnostic and therapeutic challenges.

A 22-year-old woman presented with symmetrical, well-demarcated, hyperkeratotic, erythematous plaques with a carnauba wax-like appearance on the palms (Figure 1), soles, elbows, and trunk covering approximately $5 \%$ of the body surface area. Two weeks prior to presentation, she experienced an upper respiratory tract infection without any treatment and subsequently developed redness on the palms, which became very hard and scaly. The redness then spread to the elbows, soles, and trunk. She reported itching as well as pain in areas of fissuring. Hand mobility became restricted due to thick scale.

The patient's medical history was notable for suspected psoriasis 9 years prior, but there were no records or biopsy reports that could be obtained to confirm the diagnosis. She also reported a similar skin condition in her father, which also was diagnosed as psoriasis, but this diagnosis could not be verified.

Although the morphology of the lesions was most consistent with localized PRP, atypical psoriasis, palmoplantar keratoderma (PPK), and erythroderma progressive symmetrica (EPS) also were considered given the personal and family history of suspected psoriasis. A biopsy could not be obtained due to an insurance issue. She was started on clobetasol cream $0.05 \%$ and ointment. At 2-week follow-up, her condition remained unchanged. Empiric systemic treatment was discussed, which would potentially work for diagnoses of both PRP and psoriasis. Due to the history of psoriasis and level of discomfort, cyclosporine $300 \mathrm{mg}$ once daily was started to gain rapid control of the disease. Methotrexate also was considered due to its efficacy and economic considerations but was not selected due to patient concerns about the medication.

After 10 weeks of cyclosporine treatment, our patient showed some improvement of the skin with decreased scale and flattening of plaques but not complete resolution. At this point, a biopsy was able to be obtained with prior authorization. A 4-mm punch biopsy of the right

Dr. Abrouk is from the School of Medicine, University of California, Irvine. Drs. Nakamura, Koo, and Bhutani are from the Department of Dermatology, Psoriasis and Skin Treatment Center, University of California, San Francisco. Dr. Zhu is from the Keck School of Medicine, University of Southern California, Los Angeles. Dr. Farahnik is from the College of Medicine, University of Vermont, Burlington.

The authors report no conflict of interest.

Correspondence: Michael Abrouk, MD, 515 Spruce St, San Francisco CA, 94118 (michaelabrouk1@gmail.com). doi: 10.12788 /cutis.0372 

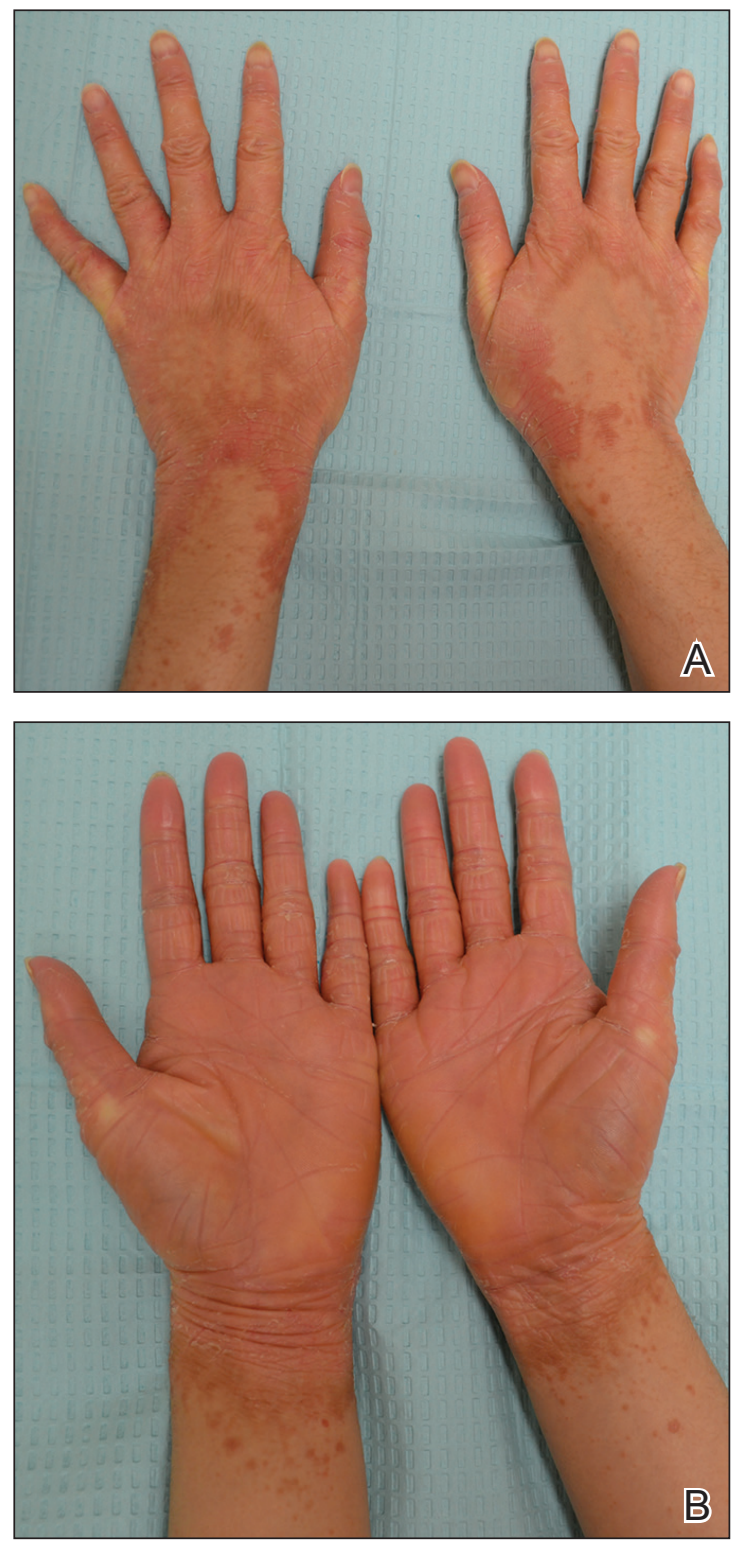

FIGURE 1. A and B, Pityriasis rubra pilaris on the hands before treatment.

flank demonstrated a psoriasiform and papillated epidermis with multifocally capped, compact parakeratosis and minimal lymphocytic infiltrate consistent with PRP. Although EPS also was on the histologic differential, clinical history was more consistent with a diagnosis of PRP. There was some minimal improvement with cyclosporine, but with the diagnosis of PRP confirmed, a systemic retinoid became the treatment of choice. Although acitretin is the preferred treatment for PRP, given that pregnancy would be contraindicated during and for 3 years following acitretin therapy, a trial of isotretinoin $40 \mathrm{mg}$ once daily was started due to its shorter half-life compared to acitretin and was continued for 3 months (Figure 2). ${ }^{6,7}$
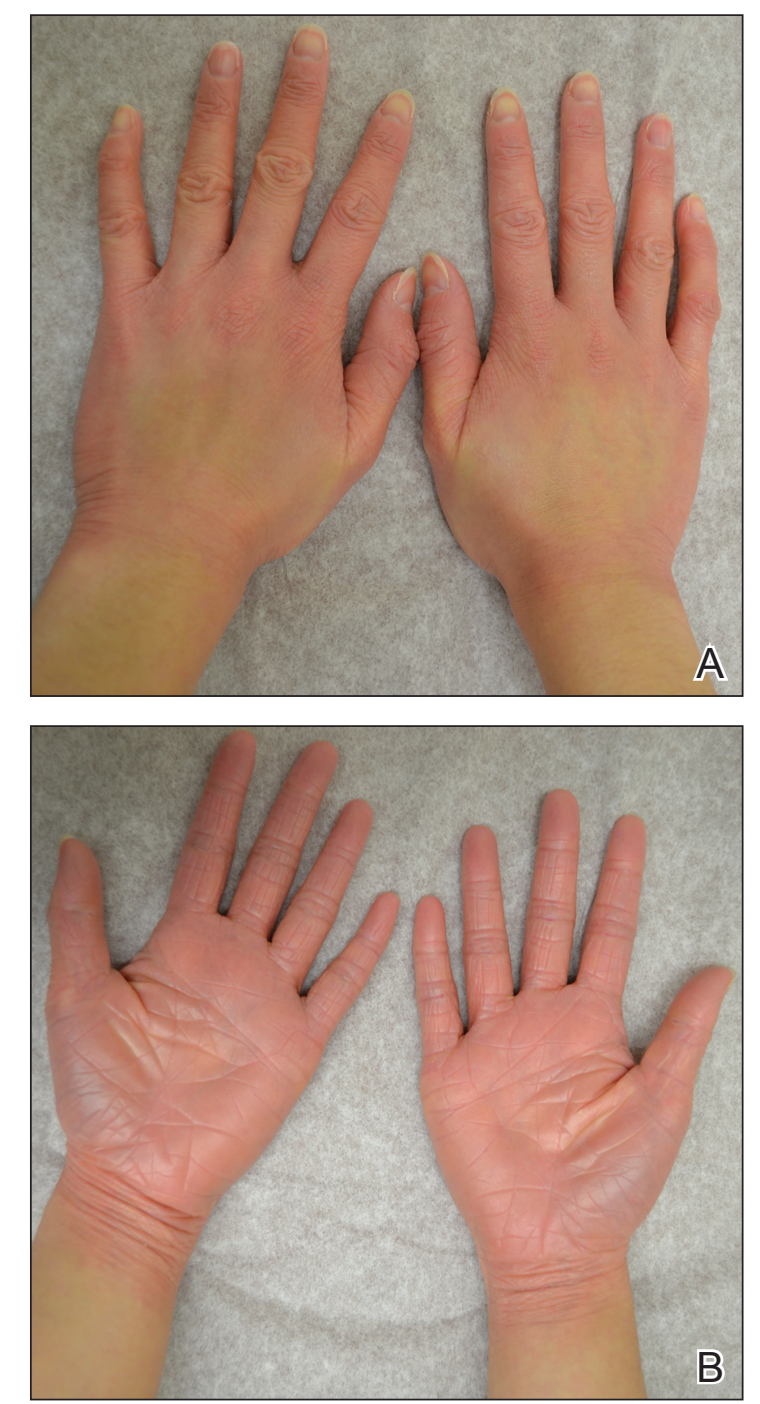

FIGURE 2. $A$ and $B$, The hands after treatment with cyclosporine $300 \mathrm{mg}$ daily for 10 weeks, followed by isotretinoin $40 \mathrm{mg}$ daily for 3 months.

The diagnosis of PRP often can be challenging given the variety of clinical presentations. This case was an atypical presentation of PRP with several learning points, as our patient's condition did not fit perfectly into any of the 6 types of PRP. The age of onset was atypical at 22 years old. Pityriasis rubra pilaris typically presents with a bimodal age distribution, appearing either in the first decade or the fifth to sixth decades of life. ${ }^{3,8}$ Her clinical presentation was atypical for adult-onset types I and II, which typically present with cephalocaudal progression or ichthyosiform dermatitis, respectively. Her presentation also was atypical for juvenile onset in types III, IV, and $\mathrm{V}$, which tend to present in younger children and with different physical examination findings. ${ }^{3,8}$

The morphology of our patient's lesions also was atypical for PRP, PPK, EPS, and psoriasis. The clinical presentation had features of these entities with 
erythema, fissuring, xerosis, carnauba wax-like appearance, symmetric scale, and well-demarcated plaques. Although these findings are not mutually exclusive, their combined presentation is atypical. Coupled with the ambiguous family history of similar skin disease in the patient's father, the discussion of genodermatoses, particularly PPK, further confounded the diagnosis. ${ }^{4,9}$ When evaluating for PRP, especially with any family history of skin conditions, genodermatoses should be considered. Furthermore, our patient's remote and unverifiable history of psoriasis serves as a cautionary reminder that prior diagnoses and medical history always should be reasonably scrutinized. Additionally, a drug-induced PRP eruption also should be considered. Although our patient received no medical treatment for the upper respiratory tract infection prior to the onset of PRP, there have been several reports of drug-induced PRP. ${ }^{10-12}$

The therapeutic challenge in this case is one that often is encountered in clinical practice. The health care system often may pose a barrier to diagnosis by inhibiting particular services required for adequate patient care. For our patient, diagnosis was delayed by several weeks due to difficulties obtaining a diagnostic skin biopsy. When faced with challenges from health care infrastructure, creativity with treatment options, such as finding an empiric treatment option (cyclosporine in this case), must be considered.

Systemic retinoids have been found to be efficacious treatment options for PRP, but when dealing with a woman of reproductive age, reproductive preferences must be discussed before identifying an appropriate treatment regimen. ${ }^{1,13-15}$ The half-life of acitretin compared to isotretinoin is 2 days vs 22 hours. ${ }^{6,16}$ With alcohol consumption, acitretin can be metabolized to etretinate, which has a half-life of 120 days. ${ }^{17}$ In our patient, isotretinoin was a more manageable option to allow for greater reproductive freedom upon treatment completion.

\section{REFERENCES}

1. Klein A, Landthaler M, Karrer S. Pityriasis rubra pilaris: a review of diagnosis and treatment. Am J Clin Dermatol. 2010;11:157-170.

2. Shenefelt PD. Pityriasis rubra pilaris. Medscape website. Updated September 11, 2020. Accessed September 28, 2021. https://reference .medscape.com/article/1107742-overview

3. Griffiths WA. Pityriasis rubra pilaris. Clin Exp Dermatol. 1980;5:105-112.

4. Itin PH, Lautenschlager S. Palmoplantar keratoderma and associated syndromes. Semin Dermatol. 1995;14:152-161.

5. Guidelines of care for psoriasis. Committee on Guidelines of Care. Task Force on Psoriasis. J Am Acad Dermatol. 1993;28:632-637.

6. Larsen FG, Jakobsen P, Eriksen $\mathrm{H}$, et al. The pharmacokinetics of acitretin and its 13-cis-metabolite in psoriatic patients. J Clin Pharmacol. 1991;31:477-483

7. Layton A. The use of isotretinoin in acne. Dermatoendocrinol. 2009; 1:162-169.

8. Sørensen KB, Thestrup-Pedersen K. Pityriasis rubra pilaris: a retrospective analysis of 43 patients. Acta Derm Venereol. 1999;79:405-406.

9. Lucker GP, Van de Kerkhof PC, Steijlen PM. The hereditary palmoplantar keratoses: an updated review and classification. Br J Dermatol. 1994;131:1-14.

10. Cutaneous reactions to labetalol. Br Med J. 1978;1:987.

11. Plana A, Carrascosa JM, Vilavella M. Pityriasis rubra pilaris-like reaction induced by imatinib. Clin Exp Dermatol. 2013;38:520-522.

12. Gajinov ZT, Matć MB, Duran VD, et al. Drug-related pityriasis rubra pilaris with acantholysis. Vojnosanit Pregl. 2013;70:871-873.

13. Clayton BD, Jorizzo JL, Hitchcock MG, et al. Adult pityriasis rubra pilaris: a 10-year case series. J Am Acad Dermatol. 1997;36:959-964.

14. Cohen PR, Prystowsky JH. Pityriasis rubra pilaris: a review of diagnosis and treatment. J Am Acad Dermatol. 1989;20:801-807.

15. Dicken CH. Isotretinoin treatment of pityriasis rubra pilaris. J Am Acad Dermatol. 1987;16(2 pt 1):297-301.

16. Layton A. The use of isotretinoin in acne. Dermatoendocrinol. 2009;1:162-169.

17. Grønhøj Larsen F, Steinkjer B, Jakobsen P, et al. Acitretin is converted to etretinate only during concomitant alcohol intake. Br J Dermatol. 2000;143:1164-1169. 